\title{
VECTOR VALUED MEASURES OF BOUNDED MEAN OSCILLATION
}

\author{
OSCAR BLASCO*
}

\section{0 . Introduction}

The duality between $H^{2}$ and $B M O$, the space of functions of bounded mean oscillation (see [JN]), was first proved by C. Fefferman (see [F], [FS]) and then other proofs of it were obtained. Using the atomic decomposition approach ([C], [L]) the author studied the problem of characterizing the dual space of $H^{1}$ of vector-valued functions. In [B2] the author showed, for the case $\Omega=\{|z|=1\}$, that the expected duality result $H^{3}-B M O$ holds in the vector valued setting if and only if $X^{*}$ has the Radon-Nikodym property. If we want to get a duality result valid for all Banach spaces we may consider vector valued measures (see [BT], where the vector valued $L_{p}$ case is treated, for an explanation) and therefore to deal with the general case it was necessary to consider a new space of vector valued measures closely related to $B M O$ (see [B1]).

In this paper we shall study such space in little more detail and we shall consider the $H^{1}-B M O$ duality for vector-valued functions in the more general setting of spaces of homogeneous type (see [CW]).

Throughout the paper $X$ will stand for a Banach space, $\Omega$ will be a space of homogeneous type (see definition in the preliminary section) and we write $L_{p}(\Omega, X)$ for the space of measurable functions on $\Omega$ with values in $X$ such that $\|f(x)\|$ belongs to $L_{p}(\Omega)$. As usual $C$ will denote a constant not necessarily the same at each occurrence.

\section{Preliminaries}

A space of homogeneous type $\Omega$ is a topological space endowed with a Borel measure $m$ and a quasi-distance $d$, that is $d: X \times X \rightarrow \mathbf{R}^{+}$with

a)

$$
d(x, y)=d(y, x),
$$

b)

$$
d(x, y)=0 \quad \text { if and only if } \quad x=y
$$

c)

$$
d(x, y) \leq K(d(x, z)+d(z, y)) .
$$

*Partially suported by the grant C.A.I.C.Y.T. PB85-0338 
and we assume that the balls $B_{r}(x)=\{y \in \Omega: d(x, y)<r\}$ form a basis of open neighborhoods of the point $x$ and there exists a constant $A$ satisfying

$$
m\left(B_{r}(x)\right) \leq A m\left(B_{r / 2}(x)\right)
$$

From (1.0) we can assume that $0<m(B)<\infty$ for every ball $B$ (otherwise $m$ would be identicaliy 0 or $\infty$ ) and therefore $m$ is a $\sigma$-finite measure on $\Omega$. Denote by $\Sigma_{0}$ the ring of bounded measurable sets. The $\sigma$-finiteness condition implies that the $\sigma$-algebra generated by $\Sigma_{0}$ coincides with the Borel $\sigma$-algebra that we shall denote by $\Sigma$.

Let us now recall the notion of atom with values in $X$. Given $1<p \leq \infty$, a function $a$ in $L_{p}(\Omega, X)$ is called $(X, p)$-atom if

a) the support is contained in a ball $B=B_{r}\left(x_{o}\right)$

b)

$$
\left(\frac{1}{m(B)} \int_{B}\|a(x)\|^{p} d m(x)\right)^{1 / p} \leq \frac{1}{m(B)} \quad(p<\infty)
$$

b')

$$
\|a(x)\| \leq \frac{1}{m(B)} \quad m-\text { a.e. } \quad(p=\infty)
$$

c)

$$
\int_{B} a(x) d m(x)=0
$$

In the case $m(\Omega)<\infty$ the constant function $\frac{1}{m(\Omega)} b$, where $b \in X$ with $\|b\|=1$, is also considered as a $(X, p)$-atom.

Note that the atoms are in the unit ball of $L_{1}(\Omega, X)$.

Following [CW] we define $H_{p}^{1}(\Omega, X)$ as the space of functions $f$ in $L_{1}(\Omega, X)$ adnitting an atomic decomposition

$$
f=\sum_{j=0}^{\infty} \lambda_{j} a_{j}
$$

where the $a_{j}$ 's are $(X, p)$-atoms and $\sum_{j=0}^{\infty}\left|\lambda_{j}\right|<\infty$. (The convergence of (1.1) is taken in $L_{1}(\Omega, X)$ ).

We get a Banach space if we consider the norm

$$
\|f\|_{H_{p}^{1}}=\inf \sum_{j=0}^{\infty}\left|\lambda_{j}\right|
$$

where the infimum is taken over all representations $f=\sum_{j=0}^{\infty} \lambda_{j} a_{j}$. 
The same arguments as in [CW] show that, in fact, for $1<p, r \leq \infty$

$$
H_{p}^{1}(\Omega, X)=H_{r}^{1}(\Omega, X) \text { (with equivalent norms). }
$$

Let us also recall the definition of vector-valued BMO. Let $1 \leq q<\infty$, an $X$ valued function which is locally in $L_{q}(\Omega, X)$ is said to belong to $B M O_{q}(\Omega, X)$ provided that

$$
\sup _{\text {ballB }}\left(\frac{1}{m(B)} \int_{B}\left\|g(x)-g_{B}\right\|^{q} d m(x)\right)^{3 / q} \leq C
$$

where $g_{B}=\frac{1}{m(B)} f_{B} g(x) d m(x)$.

Let us denote by

$$
\|g\|_{*, q}=\sup \left\{\left(\frac{1}{m(B)} \int_{B}\left\|g(x)-g_{B}\right\|^{q} d m(x)\right)^{1 / q}: \mathrm{B} \text { ball }\right\}
$$

When $m(\Omega)=\infty$ then $\|g\|_{B M O_{q}}=\|g\|_{*, q}$ gives a norm on the set of equivalence classes of functions which differ by a constant in $X$.

For $m(\Omega)<\infty$ we consider the norm $\|g\|_{B M O_{1}}=\|g\|_{*_{, q}}+\left\|\int_{\Omega \Omega} g(x) d m(x)\right\|$.

Let us recall now a few definitions about vector-valued measures we shall use later on. Let $(\Omega, \Sigma, m)$ be any $\sigma$-finite measure space, $A$ a measurable set and $1<p<\infty$. Given a vector valued measure $G$, we denote by $|G|$ the variation of $G$, that is

$$
|G|(A)=\sup \left\{\sum_{i=1}^{n}\left\|G\left(E_{i}\right)\right\|:\left(E_{i}\right) \text { partition of } \mathrm{A}\right\}
$$

and by $|G|_{p}(A)$ the $p$-variation on $A$, that is

$$
|G|_{p}(A)=\sup \left\{\left(\sum_{i=1}^{n} \frac{\left\|G\left(E_{i}\right)\right\|^{p}}{m\left(E_{i}\right)^{p-1}}\right)^{1 / p}\right\}
$$

where the supremum is taken over all finite partitions $\left(E_{i}\right)$ of disjoint measurables sets contained in $A$ with $m\left(E_{i}\right)>0$.

For the case $p=\infty$ we shall denote by $V^{\infty}(\Omega, X)$ the spacc of $X$-valued measures $G$ satisfying

$$
\|G(E)\| \leq C m(E) \text { for all measurable set } \mathrm{E}
$$

Defining the norm by the infimum of the constants satisfying (1.6) we get a Banach space.

Remark 1.1. It is not hard to see that in fact $\left\|G\left(E_{i}\right)\right\|$ can be replaced by $|G|\left(E_{i}\right)$ in the definition of $p$-variation. (See Lemma 1 in [B3])

Remark 1.2. If $G$ is a vector valued measure defined on $\Sigma_{0}$ which is absolutely continuous with respect to $m$, that is $\lim _{m(E) \rightarrow 0} G(E)=0$, then it can be extended to a measure on $\Sigma$, being still absolutely continuous with respect to m. (See [D],[DU])

We refer the reader to ([DU], [D]) and to ([J], [GC-RF]) for general theory and the properties we shall use abont vector valued measures and Hardy spaces respectively. 


\section{Vector valued measures of bounded mean oscillation}

Defnition 2.1. Let $1 \leq q<\infty$. Given a countably additive measure $G$ defined on $\Sigma$ and with values in $X$, it is said that $G$ belongs to $M B M O_{q}(\Omega, X)$ if

$$
|G|_{*, q}=\sup \left\{\left(\sum_{i=1}^{n}\left\|\frac{G\left(E_{i}\right)}{m\left(E_{i}\right)}-\frac{G(B)}{m(B)}\right\|^{q} \frac{m\left(E_{i}\right)}{m(B)}\right)^{1 / q}\right\}<\infty
$$

where the supremun is taken over all balls $B$ and over all finite partitions of $B$ in pairwise disjoint measurable sets $E_{i}$ with $m\left(E_{i}\right)>0$.

When $m(\Omega)=\infty$ then $\|G\|_{M B M O_{4}}=|G|_{*, q}$ gives a norm on the set of equivalence classes of measures: $G_{1} \sim G_{2}$ if there is $b$ in $X$ such that $G_{1}(E)-$ $G_{2}(E)=b m(E)$ for all measurable set $E$.

For $m(\Omega)<\infty$ we consider the norm $\|G\|_{M B M O_{*}}=|G|_{*, q}+\|G(\Omega)\|$.

It is obvious that if $1<q_{1}<q_{2}<\infty$ then

$$
V^{\infty}(\Omega, X) \subset M B M O_{q_{2}}(\Omega, X) \subset M B M O_{q_{1}}(\Omega, X)
$$

Remark 2.1. Let us assume $G$ belong to $M B M O_{q}(\Omega, X)$. Given a ball $B$ and a measurable set $E \subset B$, it is quite immediate to find a constant $C_{B}$ depending on $B$ satisfying

$$
\|G(E)\| \leq C_{B} \max \left(m(E), m(E)^{1-1 / q}\right)
$$

Suposse we consider $B_{n}=\left\{y \in \Omega: d\left(x_{0}, y\right)<n\right\}$ and denote by $G_{B_{n}}$ the measure $G$ concentrated on $B_{n}$, that is $G_{B_{n}}(E)=G\left(E \cap B_{n}\right)$. A glance at $(2.3)$ allows us to say that for any $1<q<\infty$ if $G$ belongs to $M B M O_{q}(\Omega, X)$ then $G_{B_{n}}$ are necessarily absolutely continuous with respect to $m$ and this clearly implies that also $G$ is absolutely continuous with respect to $m$. (Recall that for vector-measures on $\sigma$-algebras it suffices to check that they vanish on m-null sets).

Proposition 2.1. Let $1 \leq q<\infty, g$ be locally in $L_{q}(\Omega, X)$ and $G$ be an $X$ valued measure such that $G(E)=\int_{E} g(x) d m(x)$ for all measurable bounded set. E.

Then $g$ belongs to $B M O_{q}(\Omega, X)$ if and only if $G$ belongs to $M B M O_{q}(\Omega, X)$. Moreover $\|G\|_{M B M O_{q}}=\|g\|_{B M O_{q}}$.

Proof: Given any ball $B$, consider $G_{B}(E)=G(E \cap B)-\frac{G(B)}{m(B)} m(E \cap B)$. Observe that

$$
\sup \left\{\left(\sum_{i=1}^{n}\left\|\frac{G\left(E_{i}\right)}{m\left(E_{i}\right)}-\frac{G(B)}{m(B)}\right\|^{q} \frac{m\left(E_{i}\right)}{m(B)}\right)^{1 / q}:\left(E_{i}\right) \text { partition of } B\right\}
$$


coincides with the $q$-variation of $G_{B}$ on $\Omega$ divided by $m(B)^{1 / q}$ and $G_{B}$ is a measure represented by the function $\left(g-g_{B}\right) \chi_{B}$, that is

$$
G_{B}(E)=\int_{E}\left(g(x)-g_{B}\right) \chi_{B} d m(x) .
$$

Therefore the proposition follows from the equality between the $q$-variation and the norm in $L_{q}$ of the function which represents the incasure (see [D]).

Remark 2.2. In general it is not true that any measure in $M B M O_{q}(\Omega, X)$ is representable by a function, this depends on the Radon-Nikodym property. We refer the reader to [B1] for the case $\Omega=\{|z|=1\}$, but a similar result and proof can be established also in this general setting.

Proposition 2.2. Let $1 \leq q<\infty$. G belongs to $M B M O_{q}(\Omega, X)$ if and only if there exists a family of vectors in $X$, say $\left\{a_{B}: B\right.$ ball $\}$, such that

$$
\sup \left\{\left(\sum_{i=1}^{n}\left\|\frac{G(E i)}{m\left(E_{i}\right)}-a_{B}\right\|^{2} \frac{m(E i)}{m(B)}\right)^{1 / q}\right\}<\infty
$$

where the supremum is taken over all balls $B$ and over all finite partitions of $B$ in pairwise disjoint measurable sets $E_{i}$ with $m\left(E_{i}\right)>0$

Proof: The direct implication is obvious by taking $a_{B}=\frac{G(B)}{m(B)}$. To show the converse let us assume that we have $\left\{a_{B}: B\right.$ ball $\}$ with the above property, and notice that

$$
\left\|a_{B}-\frac{G(B)}{m(B)}\right\| \leq C
$$

for all $B$ (simply take the partition of $B$ given only by $B$ ).

Therefore for any $B$ and any partition

$$
\begin{gathered}
\left(\sum_{i=1}^{n}\left\|\frac{G\left(E_{i}\right)}{m\left(E_{i}\right)}-\frac{G(B)}{m(B)}\right\|^{q} \frac{m\left(E_{i}\right)}{m(B)}\right)^{1 / q} \leq \\
\left(\sum_{i=1}^{n}\left\|\frac{G\left(E_{i}\right)}{m\left(E_{i}\right)}-a_{B}\right\|^{q} \frac{m\left(E_{i}\right)}{m(B)}\right)^{1 / q}+\left(\sum_{i=1}^{n}\left\|a_{B}-\frac{G(B)}{m(B)}\right\|^{q} \frac{m\left(E_{i}\right)}{m(B)}\right)^{1 / q} \leq C
\end{gathered}
$$

As in the case of functions we can define an equivalent norm in $M B M O_{q}(\Omega, X)$. Let us take

$$
|G|_{*_{, g}}^{\prime}=\sup _{\text {ball } B}\left\{\inf _{a \in X} \frac{1}{m(B)^{1 / q}}|G-a m|_{q}(B)\right\} .
$$

Note that essentially the same argument as in Proposition 2.2. shows the following

$$
|G|_{*, q}^{\prime} \leq|G|_{*, q} \leq C|G|_{*, q}^{\prime}
$$


Proposition 2.3. Let $1<q<\infty$. If $G$ belongs to $M B M O_{q}(\Omega, X)$ then there exists a non negative function $\phi$ in $B M O_{q}(\Omega)$ such that

$$
|G|(E)=\int_{E} \phi(x) d m(x)
$$

Moreover $\|\phi\|_{B M O_{\uparrow}} \leq C\|G\|_{M B M O_{\uparrow}}$.

Proof: Since $G$ is countably additive and $m$-continuous then the same is true for the variation of $G,|G|$. Therefore using the Radon-Nikodym theorem there exists a non negative measurable function $\phi$ which represents the measure $|G|$. To show that $\phi$ belongs to $B M O_{g}(\Omega)$, we shall use Propositions 2.2 and 2.1. We simply have to find a family of real numbers $\left\{a_{B}\right\}$ such that

$$
\sup \left\{\left(\sum_{i=1}^{n}\left|\frac{|G|(E i)}{m(E i)}-a_{B}\right|^{q} \frac{m\left(E_{i}\right)}{m(B)}\right)^{1 / q}\right\}<\infty
$$

Take $a_{B}=\frac{\|G(B)\|}{m(B)}$, and observe that

$$
|| G\left|(E)-\frac{\|G(B)\|}{m(B)} m(E)\right| \leq\left|G-\frac{G(B)}{m(B)} m\right|(E)
$$

Then

$$
\begin{gathered}
\sup \left\{\left(\sum_{i=1}^{n}\left|\frac{|G|(E i)}{m(E i)}-\frac{\|G(B)\|}{m(B)}\right|^{q} \frac{m\left(E_{i}\right)}{m(B)}\right)^{1 / q}\right\} \leq \\
\sup \left\{\frac{1}{m(B)^{1 / q}}\left(\sum_{i=1}^{n}\left(\left|G-\frac{G(B)}{m(B)} m\right|\left(E_{i}\right)\right)^{q} m\left(E_{i}\right)^{1-q}\right)^{1 / q}\right\} \leq|G|_{*, q}
\end{gathered}
$$

The last inequality follows from Remark 1.1.

\section{The theorem and its proof}

In the sequel $1<p, q<\infty$, with $\frac{1}{p}+\frac{1}{q}=1$. In this section we shall achieve the duality result between $H_{p}^{1}(\Omega, X)$ and $M B M O_{q}\left(\Omega, X^{*}\right)$. We shall need several lemmas before we prove the result. The next result was done in [B1] for the circle and for $q=2$, and here we present a different approach which is valid for general spaces of homogeneous type. The author would like to point out that a similar and independent proof of the following lemma has been obtained by $\mathrm{T}$. Wolniewicz (personal communication). 
Lemma 3.1. Let $G$ be a measure in $M B M O_{q}(\Omega, X)$. Then for each integer $n \in \mathrm{N}$ we can find a measure $G_{n}$ in $V^{\infty}(\Omega, X)$ and a constant $C_{n}$ satisfying $\left|G_{n}\right|_{*, q} \leq C_{n}$ and such that

$$
|G|_{*, q} \leq \lim _{n \rightarrow \infty} C_{n} \leq K|G|_{*, q}
$$

$$
\lim _{n \rightarrow \infty} G_{n}(E)=G(E) \quad \text { for all measurable bounded set } E \text {. }
$$

Proof: Using Proposition 2.3 we frst get a function $\phi$ in $B M O_{q}(\Omega)$.

Denote by $\Omega_{n}=\{x \in X: \phi(x)>n\}$ and $\phi_{n}(x)=\min (1, n / \phi(x))$. Let us define now

$$
G_{n}(E)=\int_{E} \phi_{n}(x) d G(x) \quad\left(E \in \Sigma_{0}\right)
$$

Notice that

$$
\left\|G_{n}(E)\right\| \leq\left|G_{n}\right|(E) \leq \int_{E} \phi_{n}(x) d|G|(x) \leq \int_{E} \phi_{n}(x) \phi(x) d m(x) \leq n m(E)
$$

This, using Remark 1.2., allows to extend $G_{n}$ to $\Sigma$ and shows that $G_{n}$ belongs to $V^{\infty}(\Omega, X)$.

On the other hand

$$
G(E)-G_{n}(E)=\int_{E \cap \Omega_{n}}\left(1-\phi_{n}(x)\right) d G(x)
$$

Therefore if $E$ is contained in some ball $B$

$$
\left\|G(E)-G_{n}(E)\right\| \leq 2 \int_{E \cap \Omega_{n}} \phi(x) d m(x)
$$

Since $\phi \chi_{B}$ is in $L_{1}(\Omega)$ then taking limit as $n \rightarrow \infty$ shows (3.2).

From (2.6) we have finally to estimate $m(B)^{-1 / q}\left|G_{n}-a m\right|_{q}(B)$ for all balls $B$. Using (3.4) we have that for any $E \subset B$

$$
\left\|G(E)-G_{n}(E)\right\| \leq \int_{E \cap \Omega_{n}}(1-n / \phi(x)) d|G|(x)
$$

If $\|a\| \leq n$ then

$$
\left\|G(E)-G_{n}(E)\right\| \leq \int_{E \cap \Omega_{n}}(\phi(x)-n) d m(x) \leq \int_{E \cap \Omega_{n}}(\phi(x)-\|a\|) d m(x)
$$

Therefore we have

$$
\left|G_{n}-G\right|_{q}(B) \leq|G-a m|_{q}\left(B \cap \Omega_{n}\right)
$$


Though $|G|_{q}$ is not a measure for $q>1$ the $q$-variation es subadditive and therefore we get that for all $\|a\| \leq n$

$$
m(B)^{-1 / q}\left|G_{n}-a m\right|_{q}(B) \leq 2 m(B)^{-3 / q}|G-a m|_{q}(B)
$$

Denoting now by

$$
D_{\mathfrak{n}}=\sup _{\text {ballB }\|a\| \leq n} \inf _{\mid a n}\left\{m(B)^{-1 / q}|G-a m|_{q}(B)\right\}
$$

we get (3.1) for $C_{n}=2 C D_{n}$ where $C$ is the constant appearing in (2.6).

Notice that $V^{\infty}\left(\Omega, X^{*}\right)$ can be obviously identified with the dual of $L_{1}(\Omega, X)$. Indeed any measure $G$ in $V^{\infty}\left(\Omega, X^{*}\right)$ defines a functional $T_{G}$ acting on $X$-valued simple functions (which are dense in $L_{1}(\Omega, X)$ ) by the formula

$$
T_{G}\left(\sum_{i=1}^{n} a_{i} \chi E_{i}\right)=\sum_{i=1}^{n}<G\left(E_{i}\right), a_{i}>
$$

where $<,>$ means duality between $X$ and $X^{*}$.

Lemma 3.2. Let $1<p, q<\infty, \frac{1}{p}+\frac{1}{q}=1$ and $G$ belong to $V^{\infty}\left(\Omega, X^{*}\right)$. Then

$$
\left|T_{G}(f)\right| \leq C\|G\|_{M B M O_{q}}\|f\|_{H_{p}^{\prime}} \text { for all } f \text { in } H_{p}^{1}(\Omega, X) \text {. }
$$

Proof: Let us first take a "simple atom" in $H_{p}^{1}(\Omega, X)$, that is $s=\sum_{i=1}^{n} b_{i} \chi_{E_{i}}, E_{i} \subset B$ for some ball $B, \sum_{i=1}^{n} b_{i} m\left(E_{i}\right)=0$ and $\sum_{i=1}^{n}\left\|b_{i}\right\|_{X}^{p} m\left(E_{i}\right) \leq m(B)^{1-p}$.

For such an atom we can write

$$
T_{G}\left(\sum_{i=1}^{n} b_{i} \chi E_{i}\right)=\sum_{i=1}^{n}<G\left(E_{i}\right), b_{i}>=\sum_{i=1}^{n}<G\left(E_{i}\right)-\frac{G(B)}{m(B)} m\left(E_{i}\right), b_{i}>
$$

Therefore

$$
\begin{gathered}
\left|T_{G}(s\rangle\right| \leq \sum_{i=1}^{n}\left\|G\left(E_{i}\right)-\frac{G(B)}{m(B)} m\left(E_{i}\right)\right\|_{X} \cdot m\left(E_{i}\right)^{-1 / p_{m}} m\left(E_{i}\right)^{1 / p}\left\|_{i}\right\|_{X} \leq \\
\leq\left(\sum_{i=1}^{n}\left\|\frac{G\left(E_{i}\right)}{m\left(E_{i}\right)}-\frac{G(B)}{m(B)}\right\|_{X}^{q} \cdot m\left(E_{i}\right)\right)^{3 / q}\left(\sum_{i=1}^{n}\left\|b_{i}\right\|_{X}^{p} m\left(E_{i}\right)\right)^{1 / p} \leq \\
\leq\left(\sum_{i=1}^{n}\left\|\frac{G\left(E_{i}\right)}{m\left(E_{i}\right)}-\frac{G(B)}{m(B)}\right\|_{X}^{q} \cdot \frac{m\left(E_{i}\right)}{m(B)}\right)^{1 / q} \leq|G|_{*, q}
\end{gathered}
$$


For a general atom $a$ supported in $B$ in $H_{p}^{1}(\Omega, X)$ we can use approximation by simple functions in $L_{p}(\Omega, X)$, and find a sequence of simple functions $d_{k}$ supported in $B$ converging to $a$ in $L_{p}(\Omega, X)$, and take the sequence $s_{k}=\left(d_{k}-\int_{B} d_{k}(x) d m(x)\right) \chi_{B}$ which clearly also converges to $a$ in $L_{p}(\Omega, X)$. Hence $\left\|s_{k}\right\|_{p} \leq 2\|a\|_{p}$ for $k$ large enough, and therefore $s_{k} / 2$ are "simple atoms".

Using now that $T_{G}$ is continuous as operator on $L_{1}(\Omega, X)$, and that $s_{k}$ converges to $a$ in $L_{1}(\Omega, X)$, then

$$
\left|T_{G}(a)\right|=\lim _{k \rightarrow \infty}\left|T\left(s_{k}\right)\right|=2 \lim _{k \rightarrow \infty}\left|T\left(s_{k} / 2\right)\right| \leq 2|G|_{*, q}
$$

For a general function $f$, take any representation of $f$ in $H_{p}^{1}(\Omega, X)$, say $f=$ $\sum_{j=0}^{\infty} \lambda_{j} a_{j}$, where the $a_{j}$ arc $(X, p)$-atom and $\sum_{j=0}^{\infty}\left|\lambda_{j}\right|<\infty$ and notice that (3.8) follows from (3.9) and the fact that the series $f=\sum_{j=0}^{\infty} \lambda_{j} a_{j}$ is absolutely convergent in $L_{1}(\Omega, X)$ what implies that $T_{G}(f)=\sum_{j=0}^{\infty} \lambda_{j} T_{G}\left(a_{j}\right)$.

Theorem 3.1. Let $1<p, q<\infty$ and $\frac{1}{p}+\frac{1}{q}=1$. Then

$$
\left(H_{p}^{1}(\Omega, X)\right)^{*}=M B M O_{q}\left(\Omega, X^{*}\right) \text { (equivalent norms) }
$$

Proof: Let us take $G$ in $M B M O_{q}\left(\Omega, X^{*}\right)$, and define as above

$$
T_{G}\left(\sum_{i=1}^{n} b_{i} \chi_{E_{i}}\right)=\sum_{i=1}^{n}<G\left(E_{i}\right), b_{i}>
$$

From the definition of $H_{p}^{1}(\Omega, X)$ we can easily see that simple functions with support in balls are dense in the space, therefore it is enough to see that

$$
\left|T_{G}\left(\sum_{i=1}^{n} b_{i} \chi_{E_{i}}\right)\right| \leq C|G|_{*, Q}\left\|\left(\sum_{i=1}^{n} b_{i} \chi_{E_{i}}\right)\right\|_{H_{p}^{1}}
$$

To see (3.11) we first invoke Lemma 3.1 to find a sequence of measures $G_{n}$ in $V^{\infty}\left(\Omega, X^{*}\right)$, that according to (3.2) verifies $\lim _{n \rightarrow \infty} T_{G_{n}}(s)=T_{G}(s)$ for all simple function supported in a ball.

Secondly we use Lemma 3.2, together with (3.1) to get

$$
\begin{gathered}
\left|T_{G}(s)\right| \leq \lim _{n \rightarrow \infty}\left|T_{G_{n}}(s)\right| \leq C \lim _{n \rightarrow \infty}\left|G_{n}\right|_{*, q}\|s\|_{H_{p}} \leq \\
C \lim _{n \rightarrow \infty} C_{n}\|s\|_{H_{p}^{1}} \leq C|G|_{*, q}\|s\|_{H_{p}^{1}} .
\end{gathered}
$$

For the converse we shall deal first with the case $m(\Omega)<\infty$. Let us take now a functional $T$ in $\left(H_{p}^{1}(\Omega, X)\right)^{*}$. Since constant functions are also considered 
as $X$-atoms in the case of finite measure we have that $a \chi_{E} \in H_{p}^{1}(\Omega, X)$, what allows us to define the following $X^{*}$ valued measure.

$$
<G(E), a>=T\left(a \chi_{E}\right) \quad(a \in \mathrm{X})
$$

Given a ball $B$ and a partition of $B$, say $\left\{E_{i}\right\}$, of pairwise disjoint sets, using the duality $\left(l^{p}(X)\right)^{*}=l^{q}\left(X^{*}\right)$, we have

$$
\begin{gathered}
\left(\sum_{i=1}^{n}\left\|\frac{G\left(E_{i}\right)}{m\left(E_{i}\right)}-\frac{G(B)}{m(B)}\right\|_{X}^{q} \cdot \frac{m\left(E_{i}\right)}{m(B)}\right)^{1 / q}= \\
\left(\sum_{i=1}^{n}\left\|\left(\frac{G\left(E_{i}\right)}{m\left(E_{i}\right)}-\frac{G(B)}{m(B)}\right)\left(\frac{m\left(E_{i}\right)}{m(B)}\right)^{1 / q}\right\|_{X}^{q}\right)^{1 / q}= \\
\sup \left\{\sum_{i=1}^{n}<\left(\frac{G\left(E_{i}\right)}{m\left(E_{i}\right)}-\frac{G(B)}{m(B)}\right)\left(\frac{m\left(E_{i}\right)}{m(B)}\right)^{1 / q}, b_{i}>1: \sum_{i=1}^{n}\left\|b_{i}\right\|_{X}^{p}=1\right\} .
\end{gathered}
$$

On the other hand we have

$$
\begin{gathered}
\left|\sum_{i=1}^{n}<\left(\frac{G\left(E_{i}\right)}{m\left(E_{i}\right)}-\frac{G(B)}{m(B)}\right)\left(\frac{m\left(E_{i}\right)}{m(B)}\right)^{1 / q}, b_{i}>\right|= \\
\left.\frac{1}{m(B)^{1 / q}} \mid \sum_{i=1}^{n}<\frac{G\left(E_{i}\right)}{m\left(E_{i}\right)^{1 / p}}, b_{i}>-<\frac{G(B)}{m(B)}, \sum_{i=1}^{n} m\left(E_{i}\right)^{1 / q} b_{i}\right)>\mid= \\
=\frac{1}{m(B)^{1 / q}}\left|T\left(\sum_{i=1}^{n} m\left(E_{i}\right)^{-1 / p} b_{i} \chi_{E_{i}}\right)-T\left(b \chi_{B}\right)\right|
\end{gathered}
$$

where $b=\frac{1}{m(B)}\left(\sum_{i=1}^{n} m\left(E_{i}\right)^{1 / q} b_{i}\right)$.

Denote by $a=\frac{1}{2 m(B))^{1 / q}}\left(\sum_{i=1}^{n} m\left(E_{i}\right)^{-1 / p} b_{i} \chi_{E_{i}}-b \chi_{B}\right)$. It is elementary to show that if $\sum_{i=1}^{n}\left\|b_{i}\right\|_{X}^{p}=1$ then $a$ is a $(X, p)$-atom.

Therefore we obtain

$$
\left(\sum_{i=1}^{n}\left\|\frac{G\left(E_{i}\right)}{m\left(E_{i}\right)}-\frac{G(B)}{m(B)}\right\|_{X^{*}}^{q} \frac{m\left(E_{i}\right)}{m(B)}\right)^{1 / q} \leq 2|T(a)| \leq 2\|T\|
$$

This shows $|G|_{*, q} \leq 2\|T\|$. Since $T$ and $T_{G}$ coincide over simple atoms, we have $T=T_{G}$.

On the other hand

$$
\|G(\Omega)\| \leq \sup \left\{\quad\left|T\left(b \chi_{\Omega}\right)\right|:\|b\| \leq 1 \quad\right\} \leq m(\Omega)\|T\|
$$

and this finishes the proof for the finite measure case. 
Let us deal now with the case of $m(\Omega)=\infty$. Take a functional $T$ in $\left(H_{p}^{1}(\Omega, X)\right)^{*}$ and a ball $B$ in $\Omega$. Let us consider the following space

$$
L_{0}^{p}(B, X)=\left\{f \in L_{p}(\Omega, X): \text { supp } f \subset B \text { and } \int_{B} f(x) d m(x)=0\right\}
$$

The following function is an $(X, p)$-atom

$$
a(x)=\frac{f(x)}{m(B)^{1 / q}\|f\|_{p}} \text { for } f \in L_{0}^{p}(B, X) .
$$

hence

$$
\|f\|_{H_{p}^{1}} \leq m(B)^{1 / q}\|f\|_{p}
$$

and therefore

$$
\|T f\| \leq\|T\| m(B)^{1 / q}\|f\|_{p}
$$

This shows that $T$ defines a bounded functional on $L_{0}^{p}(B, X)$ and hence from the Hahn-Banach extension theorem, we get an element in the dual of $L_{p}(B, X)$. The characterization of the dual space $\left(L_{p}(B, X)\right)^{*}$ in terms of $X^{*}$-valued measures of bounded $q$-variation allows us to find a measure $G_{B}$ with values in $X^{*}$ verifying

$$
T(f)=\int_{B} f d G_{B} \quad f \in L_{0}^{p}(B, X)
$$

(Note that this measure is uniquely determined up to a measure $F(E)=$ $\xi m(E \cap B)$ for some $\left.\xi \in X^{*}\right)$. Now if we take an increasing sequence of balls converging to $\Omega$, say $B_{n}$, and we determine $G_{B_{n}}$ by the assumption $G_{B_{n}}\left(B_{1}\right)=0$, then we can construct a vector-valued measure on $\Sigma_{0}$, given by $G(E)=G_{B_{n}}(E)$ for $E \subset B_{n}$. It is clear that $G_{B_{n}}$ are absolutely continuous and hence the same is true for $G$. Now from remark 1.2 we get an extension to $\Sigma$.

$$
\left(\sum_{i=1}^{n}\left\|\frac{G\left(E_{i}\right)}{m\left(E_{i}\right)}-\frac{G(B)}{m(B)}\right\|_{X}^{q} \cdot \frac{m\left(E_{i}\right)}{m(B)}\right)^{1 / q}=\sup _{\|f\|_{p}=1}\left|\frac{1}{m(B)^{1 / q}} \int_{B} f d\left(G-\frac{G(B)}{m(B)} m\right)\right|
$$

For each $f \in L_{p}(B, X)$, consider $a=\frac{1}{2 m(B)^{1 / q}}\left(f-f_{B}\right) \chi_{B}$ and therefore

$$
\left(\sum_{i=1}^{n}\left\|\frac{G\left(E_{i}\right)}{m\left(E_{i}\right)}-\frac{G(B)}{m(B)}\right\|_{X^{*}}^{q} \frac{m\left(E_{i}\right)}{m(B)}\right)^{1 / q}=\sup _{a} 2|T(\alpha)| \leq 2\|T\|
$$

This completes the proof.

Remark 3.1. For $1<p, r<\infty$, $M B M O_{q}(\Omega, X)=M B M O_{r}(\Omega, X)$ with equivalent norms 
For dital spaces follows from the theorem and (1.1), and the general case is consequence of the embedding $X \subset X^{* *}$

\section{References}

[B1] O. Blasco, Hardy spaces of vector-valued functions: Duality, Trans. Amer. Math. Soc. 308 (1988), 495-507.

[B2] O. Blasco, On the dual space of $H_{B}^{1, \infty}$, Colloquium Math. 55 (1988), 253-259.

[B3] O. BLAsco, Boundary values of vector-valued functions in Orlicz-Hardy classes, Arch. Math. 49 (1987), 434-439.

[BT] S. BOCHNER, A.E. TAYLOR, Linear functionals on certain spaces of abstractly-valued functions, Annals of Math. 39 (1938), 913-944.

[C] R.R. CoIfMan, A real variable characterization of $H_{p}$, Studia Math. 51 (1974), 269-274.

[CW] R.R. CoIfman, G. Weiss, Extension of Hardy spaces and their use in Analysis, Bull. Amer. Math. Soc. 83 (1977), 569-645.

[DU] J. Diestel, J.J. Uhu, Vector Measures, Amer. Math. Soc. Mathematical Surveys 15 (1977).

[D] N. Dinculeanu, "Vector measures," Pergamon Press, New York, 1967.

[F] C. Fefferman, Characterizations of bounded mean oscillation, Bull. Amer. Math. Soc. 77 (1971), 587-588.

[FS] C. Fefferman, E.M. Stein, $H_{p}$ spaces of several variables, Acta Math. 129 (1972), I37-193.

[GC-RF] J. Garcia-Cuerva, J.L. Rubio de Francla, "Weighted notm inequalites and related topics," North-Holland, Amsterdam, 1985.

[JN] F. JoHN, L. NiRENBERg, On functions of bounded mean oscillation, Comm. Pure Appl. Math. 14 (1961), 415-426.

[J] J.L. JOURNE, "Calderón-Zygmund operators, pseudo-differential operators and the Cauchy integral of Calderón," Lectures Notes in Math. 994, Springer-Vertlag, Berlin, 1983.

[L] R.H. LATTER, A characterization of $H_{p}\left(\mathbf{R}^{n}\right)$ in terms of atoms, Studia Math. 62 (1978), 93-101. 